\title{
X-ray Detection Research of Current Transformer
}

\author{
Kuo Gao \\ North China Electric Power University Yunnan \\ Power Grid Corporation Graduate workstation, \\ Yunnan Kunming, China \\ gaokuo66@163.com
}

\author{
Hong Yu, Tieqiao Guo, Erjun Pang, \\ Tao Dong, Zhiwang Cheng, Jianhui Zhang \\ High Voltage Research Institute of Yunnan Electric \\ Power Research Institute, \\ Yunnan Kunming, China, yuhong2388245@163.com
}

\begin{abstract}
Since the structure of electromagnetic current transformer is fully enclosed, When the fault occurs, using the traditional electrical insulation detection methods only detected whether it has defects or not. We cannot analysis the form and nature of the defects by using that detection method. In this paper, we use $X$-ray DR detection technology on the $35 \mathrm{kV}$ electromagnetic current transformer. After the disintegration of the device, we verify the detection result by the $X$-ray DR detection system. From the results, we can see that compare with the other electrical insulation detection methods; the X-ray DR detection technology can be more intuitive found the location of the defect.
\end{abstract}

\section{Keywords-X-ray, Current transformer, Visualization}

\section{INTRODUCTION}

The electromagnetic current transformer has been running for nearly a century, the principles and manufacturing technologies are mature. At the same time due to its closed structure it can run in the grid stability. In China electric power system, the electromagnetic current transformer that output signal is $5 \mathrm{~A}$ or $10 \mathrm{~A}$ are widely used.

The main role of the electromagnetic current transformer in the grid system is to transform the high current of primary side into the low current of secondary side so that we can measure the operating parameters of the primary side. From the measurement parameters, we can protect and control the power system. Thus, the stable and reliable operation in the power system has great significance ${ }^{[1]}$.

However, the maintenance of electromagnetic current transformer mainly uses preventive maintenance system. The preventive maintenance system is regular inspection and replacement of the current transformer equipment within the stipulated period.

Some of the traditional electrical insulation testing methods such as insulation resistance test, pressure test, and partial discharge detection can only understand the defects of the external physical parameters and the general area in which they are situated. These methods cannot locate the internal defect or malfunction of the equipment. It is not conducive to the development of maintenance strategies.

In this paper, we lead the X-ray DR detector technology into the detection field of electromagnetic current transformer. We can observe the internal structure of the device directly in the case of equipment without disassembly to discover the existence of the defect. The system can obtain the image of the device's internal structure within a few seconds. It can be used without space and environmental constraints. The system has been successfully applied in the GIS and tank circuit breakers and other substation equipment.

\section{X-RAY DR DETECTION SYSTEM}

$\mathrm{X}$-ray detection technology as an important means of industrial-ray detection is widely used in materials testing, food testing, manufacturing, medical and military, etc ${ }^{[2]}$. Xray non-destructive testing imaging technology has gone from the traditional film imaging technology, CR (Computer Radiography, CR) and DR (Digital Radiography, DR) ${ }^{[3]}$.

$\mathrm{X}$-ray DR digital imaging technology is the most advanced digital imaging technology. It using a hard flat panel detector to receive the remaining X-rays through the subject device, then converts the signal to digitized current. In the last via a data connection inside the device, the images displayed directly to the mobile workstation. For the detection of different locations, we can simply move the imaging plate without having to replace the imaging film. Compared with traditional film imaging technology and CR, X-ray DR digital imaging technology not only greatly improves the detection efficiency and due to the small size of the detection equipment, it has portable and real-time characteristics. At the same time, X-ray DR detection system has powerful image processing capabilities. It can use the conventional image processing method such as image denoising and image enhancement to optimize the image. It also has high resolution and snr. The figure below shows the $\mathrm{X}$-ray DR detection system structure diagram.
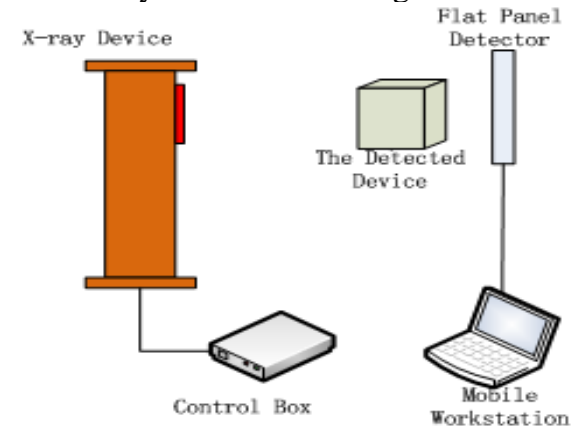

Fig.1 X-ray DR Detection System structure diagram 
When in field test, we can adjust the parameter values of the X-ray machine by the control box. These parameters include tube voltage $U$, the tube current I, the focal length F, the exposure amount $\mathrm{T}$ and collection frequency. Through adjusting these parameters, it could change the penetrating power of the X-ray and the image gray. The collected images through the flat panel detector displayed directly on the mobile workstation after processing. Finally, the collected image could be processed by using the image processing software which comes from the system. So that we could clear, intuitive observe the internal of devices ${ }^{[4-5]}$.

The electromagnetic current transformer is made up of core and winding. Its primary side and the secondary side are fully enclosed by an epoxy resin. In order to analyze the internal structure, it mainly relies on the disintegration of the current transformer. Due to the hard structure of the epoxy resin and the characteristics of the overall placement, after the disintegration it almost impossible to distinguish the correct parts of the failure. It will have impact on analyzing the form and nature of the defect.

\section{THE APPLICATION X-RAY DR DETECTION SYSTEM}

November 25, 2012. At Hong he Power Supply Bureau, The \# 1 main transformer of the Chuk Yuen substation $220 \mathrm{kV}$ has been differential protection. The breakers in $220 \mathrm{kV}$ side of 201,110 kV side of 301 have been trips.

After the failure, first, the current transformer secondary circuit is testing and found no obvious abnormalities. We suspected the line C-phase current transformer failure resulting in accidents. At the same time, there are some carbonization small particles at the base position of C-phase current transformer. Other appearance characteristics of the current transformer are abnormal. So we take the current transformer to pilot study in order to observe whether it has defects.

The current transformer is electromagnetic and the insulation is fully enclosed by epoxy. For a comprehensive analysis of the form and nature of its defects, the test complex traditional electrical insulation testing methods and $\mathrm{X}$-ray DR detection technology. We developed a detailed set inspection process. As follows:

First, we select the B-phase TA (B-phase current transformer) as a comparison to test. B-phase TA (B-phase current transformer) and C-phase TA (C-phase current transformer) are the same batches of products.

A) To measure the insulation resistance of the TA in order to observe its insulating state.

B) AC voltage test in accordance with the nameplate.

C) Excitation test. We can measure the knee voltage, knee current and resistance conversion value at $25^{\circ} \mathrm{C}$ and $27^{\circ} \mathrm{C}$.

D) PD experiments at different voltage levels at the same time using the infrared imaging test and UV imaging test to observe whether it appears discharge phenomenon and overheating.

E) Using X-ray DR detection system to observe its internal structure.

\section{A. Insulation Resistance Measurement}

The test is to measure the insulation resistance of $\mathrm{C}$ phase TA and B-phase TA. The results are as follows Table1.

TABLE I INSULATION RESISTANCE VALUE

\begin{tabular}{|c|c|c|c|}
\hline $\begin{array}{l}\text { Insulation } \\
\text { resistance } \\
\text { valu (M } \Omega)\end{array}$ & $\begin{array}{l}\text { C-phase } \\
\text { TA }\end{array}$ & \multicolumn{2}{|c|}{$\begin{array}{l}\text { B-phase } \\
\text { TA }\end{array}$} \\
\hline Primary & inding to & 0.9 & $10000+$ \\
\hline \multicolumn{4}{|c|}{ 1S, 2S and ground } \\
\hline \multicolumn{4}{|c|}{ 2S and ground } \\
\hline $\begin{array}{l}\text { 2S to Primary } \\
1 \mathrm{~S} \text { and grour }\end{array}$ & vinding, 2.1 & & $000+$ \\
\hline
\end{tabular}

Through the insulation resistance test, we found that the insulation of C-phase TA between the primary winding and the secondary winding has been breakdown. Due to the insulation has been breakdown, the AC voltage test, Excitation test and PD experiments cannot continue. In order to in-depth analysis the reason of the current transformer insulation breakdown, we use X-ray DR Detection System to detect the internal structure.

\section{B. $X$-ray DR Detection}

In order to find the difference of internal structure between fault current transformer and the normal current transformer, we respectively detect the C-phase TA and Bphase TA by using X-ray DR Detection System. Due to the structure of first outlet end cluster of parachutes is simple, the complex structure are concentrated in the windings and the core. Therefore, we only detect the position of the core and windings. We set the parameters of X-ray generating apparatus according to the table 2 .

TABle II the PARAMETERS OF CURRENT TRANSFORMER TEST

\begin{tabular}{|c|c|}
\hline $\begin{array}{l}\text { Exposure } \\
\text { Amount } \\
\text { (s) }\end{array}$ & $\begin{array}{c}\text { Collection Tube Tube The focal } \\
\text { Frequency voltage current I length } \\
\mathrm{U}(\mathrm{kV}) \quad(\mathrm{mA}) \quad(\mathrm{mm})\end{array}$ \\
\hline 2 & $\begin{array}{llll}4 & 300 & 3.0 & 750\end{array}$ \\
\hline
\end{tabular}

The current transformers are arranged according to the position shown in Figure 2. The occurrence port of X-ray machine is aligned to the core and winding of current transformer. 


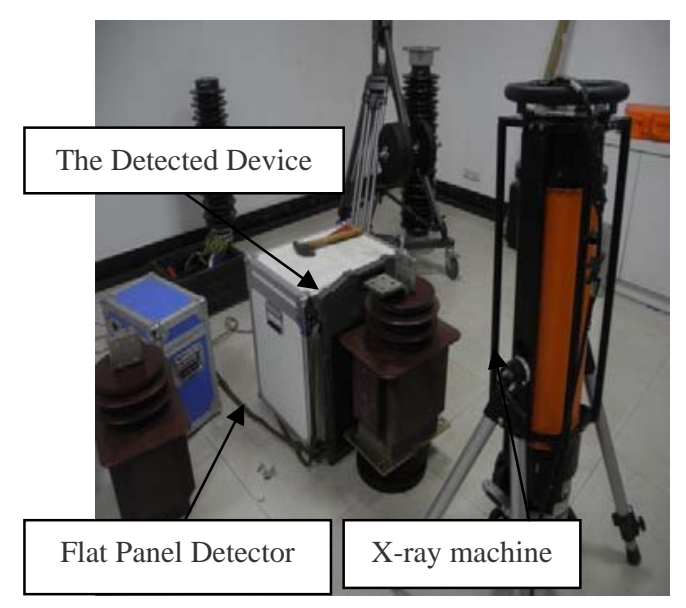

Fig.2 Equipment Layout

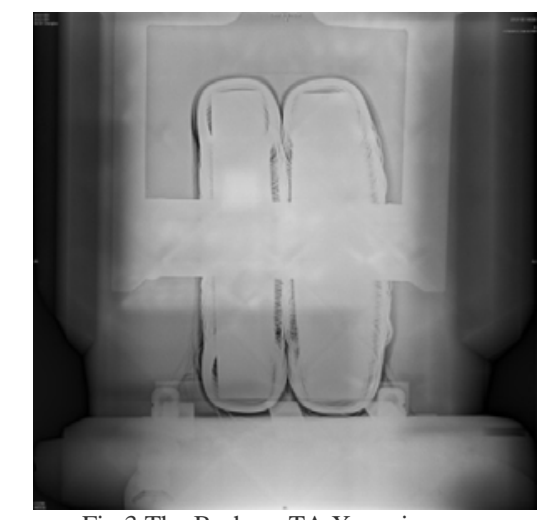

Fig.3 The B-phase TA X-ray image

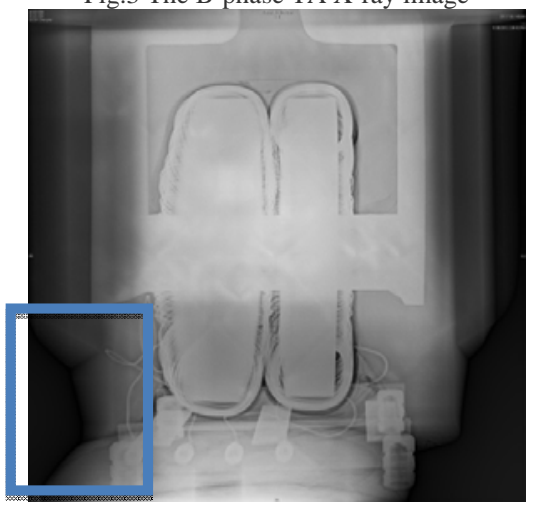

Fig.4 The C-phase TA X-ray image

Through the X-ray images, we can see that the secondary winding lead of B-phase TA is neat at the bottom of the TA and the epoxy resin casts tidily, no bubbles, impurities, etc. While, we find the secondary winding lead of C-phase TA is curve and overlapping. And the secondary winding lead of C-phase TA is too close from the first side of the copper plate. To contrast with the marked of nameplate, the position of fault is located in the 1S1 leading-out terminal of secondary terminals. Through the $\mathrm{X}$-ray image, we preliminary inferred the C-phase TA has been breakdown is due to the lead of secondary winding too close from the first side. The manufacturing process of current transformer is unsophisticated lead to the insulation breakdown. Before pouring, the secondary winding lead is not fixed or firmly fixed, resulting in the secondary lead flying out and closing from the first side. At the same, due to the secondary winding lead of C-phase TA is curve and overlapping, there will be mechanical stress in the insulating resin where have defects. Due to the presence of mechanical stress, after pouring completed, there will be a hole in the position. Once the hole is generated, During use, there will produce reactive gas (ozone, nitrogen oxides) in the hole and corrosive local insulation. This will cause unrecoverable insulation damage. With the gradual increase of the extent of corrosion, after some time of operation, the insulation properties will continue to fall leading to a sudden breakdown ${ }^{[6-7] .}$

\section{DISINTEGRATION}

In order to verify the results of X-ray detector, we disintegrate C-phase TA to observe and analysis it. Due to the hard structure of the epoxy resin and the characteristics of the overall placement, the disintegration will cause irreversible damage. The photos of disintegration are shown below.

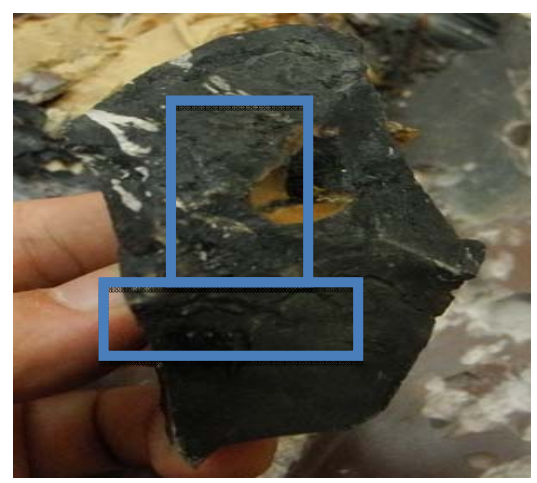

Fig. 5 the Hole and Creepage

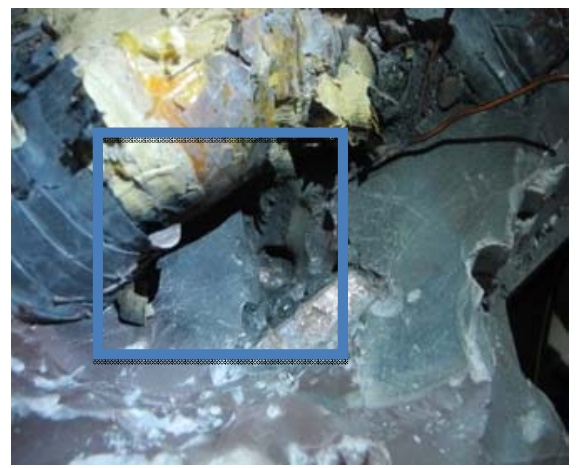

Fig.6 Primary Winding Copper Plate

In the process of disintegration, there is some odor gas overflow with burning smell. From the fig.5, the fragments are the insulating material around the secondary winding 1S1 of C-phase TA, there is obviously hole and creepage phenomenon. Around the hole, we find the epoxy resin 
become to brown and there is a carbonized conductive channel. From the fig.6, we find the primary winding of copper; this is the inflection point of a copper plate through the core. At the same time in the position we find the phenomenon of creepage. It showed that because the manufacturing process, resulting in the primary winding and the secondary winding is too close. That is why the insulation is breakdown. From the result of disintegration, we find the conclusions coincide with the results of X-ray detection. It shows that Through X-ray DR detection technology, the diagnostic results of the current transformer is accurate and effective.

\section{CONCLUSION}

In this paper, through analysis the reason of the current transformer failure, we comprehensive utilization of electrical insulation testing methods and X-ray DR detection technology. It verifies the possibility that the X-ray DR detection technology can detect the electromagnetic current transformer. The uses of the technology can accurately and rapidly diagnosis the type and nature of defects without disintegration. It provides new methods and ideas for the detection of power equipments. At the same time, the technology provides a basis for overhaul and decision support of the electric power equipments.

Currently, for the review and testing of the current transformer which accesses to the grid system mainly rely on the traditional electrical insulation testing methods such as insulation resistance test, pressure test, and partial discharge detection. These methods can quickly find out whether there is insulation abnormalities, PD exceeded and so on. And these methods can understand the defects of the external physical parameters and the general area in which they are situated. But it cannot inform of the reason, the form and the nature of defects. The X-ray DR detection can observe and analysis the internal of device in the case of nondisintegration. It can clearly shows the internal structure of device, the discovery of defects in a timely manner and discover the defects in time. Therefore, in order to guarantee the safe and stable operation of power grid, the network testing should be strengthening for the solid insulation equipment. It recommends that the X-ray DR detection should add to the routine testing. In the case of the conventional detection methods cannot detect, we use X-ray DR technology to detect. To avoid incidents, through the utilization of various detection methods, the accidents nipped in the bud stage and minimize the failure rate.

\section{REFERENCES}

[1] Gu Jianjun. The Introduction of Current transformer secondary circuit detection method. Chinese High-tech Enterprises, 2009,vol.131,no.20,pp.49-52

[2] Yu Boyuan,Mo Dawei, Zou Jianhong,Yang Youcun. A direct digital X-ray scan imaging (DR) system. China Medical Equipment, 2005, vol.2, no.4, pp.42-45

[3] Dong Xu. Medical X-ray digital photography (CR / DR) system research and evaluation of the detection method. China Medical Equipment,2010,vol.7, no.1,pp.8-11.

[4] Li Yan.CR and DR technology application of pressure equipment weld the latest international developments. Non-Destructive Testing,2009,vol.33, no.4,pp.1-6.

[5] Li Yan. New Trends in industrial DR. Non-Destructive Testing,2006, vol.30, no.6, pp.1-4.

[6] Weng Chaochen, Shang Yuan. The $35 \mathrm{kV}$ substation high-voltage current transformer several ground fault analysis. Pharmaceutical Engineering Design,2010,vol.31,no.1, pp.56-58.

[7] Li Yunfen, Pneg Fucheng, The Luodong power plant $35 \mathrm{kV}$ current transformers Dielectric Breakdown. Guangxi Electric Power Technology,2001,no.2,pp48-49. 